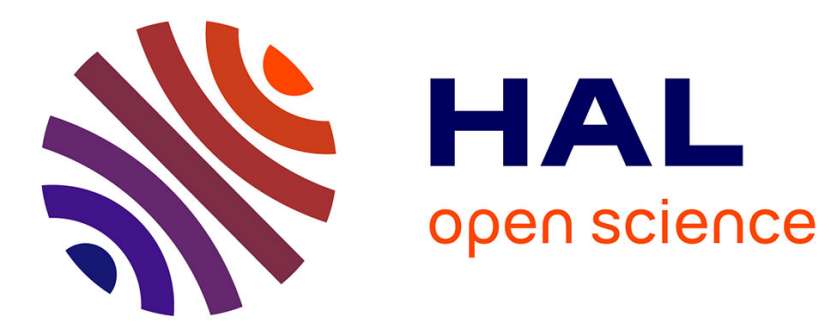

\title{
Precision Bragg reflectors obtained by molecular beam epitaxy under in situ tunable dynamic reflectometry control
}

\author{
Véronique Bardinal, R. Legros, Chantal Fontaine
}

\section{To cite this version:}

Véronique Bardinal, R. Legros, Chantal Fontaine. Precision Bragg reflectors obtained by molecular beam epitaxy under in situ tunable dynamic reflectometry control. Applied Physics Letters, 1995, 67 (23), pp.3390-3392. 10.1063/1.114903 . hal-02565592

\section{HAL Id: hal-02565592 \\ https://hal.science/hal-02565592}

Submitted on 6 May 2020

HAL is a multi-disciplinary open access archive for the deposit and dissemination of scientific research documents, whether they are published or not. The documents may come from teaching and research institutions in France or abroad, or from public or private research centers.
L'archive ouverte pluridisciplinaire HAL, est destinée au dépôt et à la diffusion de documents scientifiques de niveau recherche, publiés ou non, émanant des établissements d'enseignement et de recherche français ou étrangers, des laboratoires publics ou privés. 


\title{
Precision Bragg reflectors obtained by molecular beam epitaxy under in situ tunable dynamic reflectometry control
}

\author{
V. Bardinal, R. Legros, and C. Fontaine
}

Laboratoire d'Analyse et d'Architecture des Systèmes du Centre National de la Recherche Scientifique, 7 Avenue du Colonel Roche-31077-Toulouse Cedex-France

\begin{abstract}
Highly accurate layer thicknesses are required for multilayers involved in photonic devices, such as Bragg reflectors. In this letter, we demonstrate that precise, real-time monitoring of molecular beam epitaxy growing layers can be achieved by near-normal incidence dynamic reflectometry with a tunable sapphire-titanium laser used as a source. The advantage of this new technique lies in the possibility of synchronizing the material changes and the reflectivity extrema by selecting adequate analysis wavelengths. This technique is shown to provide $885 \mathrm{~nm}$ GaAs-AlAs Bragg reflectors with a layer thickness accuracy in excess of $1 \%$.
\end{abstract}

Distributed Bragg reflectors are key elements in optoelectronic vertical cavity devices like vertical cavity surface emitting lasers ${ }^{1}$ and spatial light modulators ${ }^{2}$ used in such applications as optical communications, optical interconnects, and optical signal processing. In particular, (Ga,Al)As mirrors are attractive, because GaAs and AlAs are lattice matched and exhibit highly different optical indices. More- over, non-absorbing Bragg reflectors can be obtained for optical communications wavelengths. Vertical cavity devices are multilayer structures, which were found to be highly sensitive to the thickness and composition of their unit layers. ${ }^{1,2}$ In particular, these errors are detrimental for the Bragg reflectors to be used as elements in vertical cavity devices be- cause the reflector calculation wavelength has to be 
precisely tuned to the device resonance wavelength within a very tight tolerance. To meet these stringent growth requirements, several in situ thickness monitoring techniques have been developed: reflection high-energy electron diffraction; ${ }^{3}$ flux monitoring, ${ }^{4}$ spectroscopic ellipsometry; ${ }^{5}$ interferometric pyrometry; ${ }^{6}$ and dynamic optical reflectometry (DOR). The latter was initially developed with fixed single-wavelength laser light sources: He-Ne lasers $(633 \mathrm{~nm}),{ }^{6}$ laser diodes $(670 \mathrm{~nm}),{ }^{7}$ or argon $(514$ $\mathrm{nm}$ ) lasers. ${ }^{8}$ More recently, the DOR monitoring accuracy has been improved by the use of broadband sources. ${ }^{9}$ In a previous letter, ${ }^{10}$ the refractive index dispersion at high temperature measured by DOR with a tunable sapphire-titanium laser used as the light source has been reported by the authors. In this letter, it is shown that this extended DOR technique, called "tunable dynamic optical reflectometry" (TDOR), can monitor molecular beam epitaxial (MBE) growth of GaAs-AlAs Bragg reflectors, when associated with an accurate modeling of the reflectivity changes during growth. This technique leads to highly accurate multilayer structures because MBE growth can be monitored under optimized conditions.

The Bragg reflectors designed for this study are centered at $885 \mathrm{~nm}$ at room temperature, and consist of a stack of ten periods of GaAs and AlAs alternating layers with quarter-wave thicknesses of 62.3 and $74.3 \mathrm{~nm}$, respectively. These multilayers were grown by MBE in a 3200P Riber system, specially designed for optical in situ monitoring: a viewport is located such that the optical analysis can be performed in the optimal growth geometry which allows high thickness uniformity to be achieved $(<1 \%$ over 2 in. diameter). Growth details are given elsewhere. ${ }^{10} \mathrm{~A}$ systematic oxide desorption procedure was applied to reproducibly calibrate growth temperature at $600^{\circ} \mathrm{C}$, while $\mathrm{Ga} / \mathrm{As}$ and $\mathrm{Al} / \mathrm{As}$ flux ratios were initially optimized by reflection high-energy electron diffraction oscillations. Growth rates of about 1 $\mu \mathrm{m} / \mathrm{h}$ were used for both binaries.

For TDOR monitoring, ${ }^{10}$ a sapphire-titanium laser beam illuminates via an optical fiber the growing layer surface at nearly normal incidence $\left(2^{\circ}\right)$. Then, after being reflected, it is detected by a Si photodiode. The signal at the lock-in amplifier output is acquired by a computer also used for laser wavelength tuning via a stepper motor. The wavelength range used for $885 \mathrm{~nm}$ Bragg reflector monitoring will be shown to be comprised between 900 and $970 \mathrm{~nm}$. Normal-incidence dynamic reflectance growth monitoring consists of recording the interferences of a light beam reflected from the surface and interfaces of a 
growing multilayer. As long as growth proceeds, reflectivity evolves with the increase in the uppermost layer thickness. Modeling of reflectivity along growth greatly helps understand the key features of the reflectivity changes. Generally, the optical behavior of a multilayer is modeled through use of the Abelès matrix formalism, ${ }^{11}$ which describes the optical properties of a layer in terms of a characteristic matrix which takes into account layer optical indices and thickness, light wavelength, and incidence angle. A multilayer stack can be represented as an equivalent layer whose matrix is the product of the characteristic matrices of the successive layers in the stack, ${ }^{12,13}$ thereby allowing immediate calculation of the reflectance of the multilayer structure. This formalism allows the already grown multilayer fraction to be considered as an equivalent material; thus whatever the materials underneath, they will behave as a single substrate with a single layer growing onto it. During growth of a thick layer, the calculated DOR signal exhibits an oscillator behavior. ${ }^{10}$ For transparent materials, all oscillations have the same period equal to $\lambda / 2 n$. For absorbing materials, the first oscillation is shorter than the following ones, because of interfacial dephasing. The latter is all the more important as the absorbing material is thick. Then substrate absorption is of the utmost importance.

The layers of the $885 \mathrm{~nm}$ Bragg reflectors are only slightly thicker than the quarter-wave thicknesses of the standard DOR monitoring wavelengths: 633 (Ref. 6), 670 (Ref. 7), or $514 \mathrm{~nm}$ (Ref. 8). In this case, modeling shows the complex oscillatory behavior reflectivity changes along growth, the significant shape being given in Fig. 1(a). Such a variation makes precise control of the growing layer thick- ness, by standard DOR difficult, as the material changes do not correspond to identifiable reflectivity changes. Indeed, with this technique the only possibility of real-time monitoring would lie in the presence of critical points in the oscillation shapes when MBE cell shutters have to be actuated be- cause the expected thickness for the growing material has been reached and the next layer growth has to be initiated. To do this, the reflectivity extrema appear well suited because they can be anticipated by studying the derivative time variation, these critical reflectivity points corresponding to a derivative value of zero. Under these conditions, reflectivity changes along the multilayer growth would exhibit an oscillatory variation, synchronized with the growth of alternating layers, as shown in Fig. 1 (b).

To investigate this promising extended DOR technique, those wavelengths that allow the DOR to be optimized for multilayer growth monitoring were calculated using Abelès modeling. The geometry of 
the multilayer to be grown was known at room temperature. Nonetheless, it had to be considered at the growth temperature, $600^{\circ} \mathrm{C}$, used when TDOR would be monitored. Here, the temperature dependence of all the parameters involved in the calculation had to be taken into account. On the one hand, lattice expansion with temperature was calculated to get the layer thicknesses at the growth temperature. On the other hand, dispersion of the GaAs and AlAs optical indices, at that temperature, had to be determined. Refractive index dispersion for these two binaries had already been determined by TDOR prior to this study in the 750-1000 nm wavelength range of the sapphire:titanium laser. ${ }^{10}$ Conversely, the absorption coefficient dispersion which had not yet been characterized was expected to be significant for GaAs, whose gap is shifted to $1.092 \mu \mathrm{m}$ when temperature rises to $600{ }^{\circ} \mathrm{C} .{ }^{14}$ The absorption index $(\kappa)$ of a growing layer can be measured from the reflectivity oscillation dampening observed to occur during layer growth. ${ }^{15}$ For this study, intermediate AlAs layers were grown prior to GaAs growths in order to get GaAs reflectivity oscillations..$^{10}$ As expected, no significant absorption was observed for AlAs. Dampening of the GaAs reflectivity oscillations was measured for some wavelengths as growth proceeded. The $\kappa$ values derived were $0.125,0.12,0.11$, and 0.105 for $900,920,940$, and $960 \mathrm{~nm}$, respectively. These values were com- pared with those obtained by extrapolating Yao's results. ${ }^{16}$ Our experimental values were found to be in the same range, whereas the dispersion law appears slightly different.

Following determination of these parameters, the optimal wavelengths to be required for DOR monitoring of the Bragg reflector under study were computed. A wavelength was calculated for each layer, thereby providing a reflectivity extremum for the final layer thickness. The result is shown in Fig. 2. As can be seen, the wavelengths were found to evolve along growth. The wavelengths required for TDOR monitoring of $885 \mathrm{~nm}$ Bragg reflectors ranges from 900 to $970 \mathrm{~nm}$, and, as expected, are well suited to the use of a sapphire-titanium laser. Their sharp initial decrease originates from the GaAs substrate absorption which causes a significant interfacial dephasing. As soon as some alternating layers are grown, the substrate effect decreases and wavelengths quickly tend to an asymptotic value. The latter corresponds to that of the reflector which progressively is formed during growth as evidenced by the progressive increase in the reflectivity maxima towards 1 in Fig. 1(b). This multilayer, an exact Bragg reflector at room temperature, is now an asymmetrical reflector at $600{ }^{\circ} \mathrm{C} .{ }^{17}$ Indeed, layer thicknesses are 
no longer $\lambda / 4 n$ as a result of the difference in GaAs and AlAs refractive index temperature dependence.

These optimal TDOR monitoring wavelengths were used to grow $885 \mathrm{~nm}$ GaAs-AlAs reflectors. Growth was performed under TDOR monitoring; at the beginning, the laser was initially tuned to the initial wavelength calculated for monitoring the first layer thickness. Afterwards, the cell shutters were alternatively opened and closed every time the reflectivity derivative was observed to reach 0 . Simultaneously, the laser was tuned to the next calculated wavelength in order to provide the optimal monitoring of the new layer being grown. The reflectivity spectrum of the grown Bragg reflector at room temperature is given in Fig. 3. The calculated spectrum is also plotted for comparison purposes. As expected, a good agreement is obtained between both spectra, indicating a thickness precision in excess of $1 \%$.

To conclude, we have presented a new dynamic optical reflectometry technique based on the use of a tunable laser which allows real-time monitoring of layer thicknesses grown by MBE. It has resulted in a precise monitoring $(<1 \%)$ of thicknesses by synchronizing the change in material with reflectivity extrema. This technique should be of great interest for monitoring the growth of all multilayer structures used in optoelectronics devices where stringent requirements are imposed on multilayer geometry. Finally, it should be noted that TDOR paves the way for complete MBE growth automation, as it makes it possible to drive cell shutters changes from reflectivity analysis.

\section{Acknowledgements}

The authors gratefully acknowledge G. Lacoste for technical assistance and A. Muñoz-Yagüe for fruitful discussions.

\section{References}

1 R. D. Dupuy, D. G. Deppe, C. J. Pinzone, N. D. Gerrard, S. Singh, G. J. Zydzik, J. P. Van der Ziel, and C. A. Green, J. Cryst. Growth 107, 790 (1991).

2 K. K. Law, J. L. Merz, and L. A. Coldren, J. Appl. Phys. 72, 855 (1992).

3 J. D. Walker, K. Malloy, and S. Wang, IEEE Photonics Technol. Lett. 2, 162 (1990). 
4 S. A. Chalmers, K. P. Killeen, and E. D. Jones, Appl. Phys. Lett. 65, 4 (1994).

5 D. E. Aspnes, W. E. Quinn, and S. Gregory, Appl. Phys. Lett. 57, 2707(1990).

6 F. G. Göbel and H. Möller, J. Vac. Sci. Technol. B 12, 207 (1994).

7 T. Farrell and J. V. Armstrong, Appl. Surf. Sci. 86, 582 (1995).

8 Y. Rafflé, R. Kuszelewicz, R. Azoulay, G. L. Roux, J. C. Michel, L. Dugrand, and E. Toussaere, Appl. Phys. Lett. 63, 3479 (1993).

${ }^{9}$ K. P. Killeen and W. G. Breiland, J. Electron. Mater. 23, 179 (1993).

10 V. Bardinal, R. Legros, and C. Fontaine, Appl. Phys. Lett. 67, 244 (1995).

11 F. Abelès, Ann. Phys. Paris 5, 596 (1950).

12 H. A. MacLeod, Thin-Film Optical Filters, 2nd ed. (Adam Hilger, Bristol, 1986).

13 M. Born and E. Wolf, Principles of Optics, 6th ed. (Pergamon, New York, 1988), Chap. 1.

14 Y. P. Varshni, Physica (Netherlands) 34, 149 (1969).

15 H. Sitter, G. J. Glanner, and M. A. Herman, Vacuum 46, 69 (1995).

16 H. Yao, P. G. Snyder, and J. A. Woollam, J. Appl. Phys. 70, 3261 (1991).

17 C. Fontaine, P. Requena, and A. Muñoz Yagüe, J. Appl. Phys. 68, 5366 (1990). 
a)

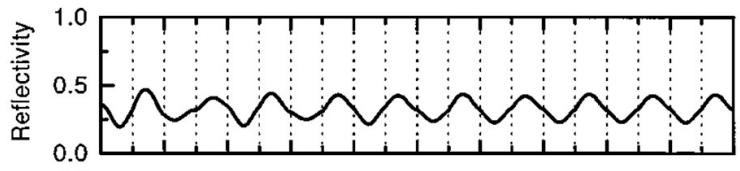

b)

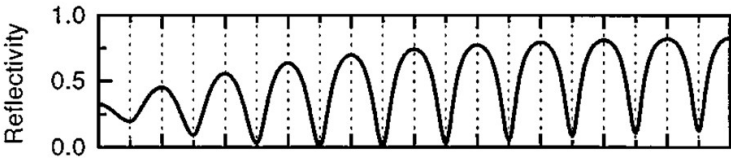

c)

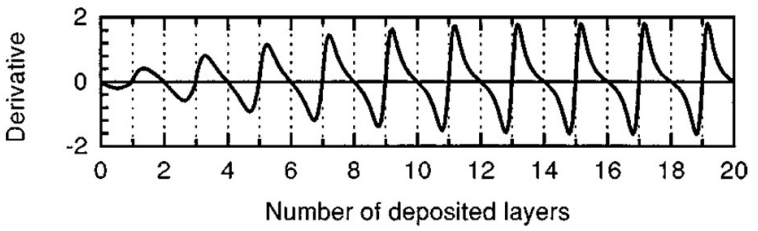

FIG. 1. Reflectivity variation during $600^{\circ} \mathrm{C}$ growth of a Bragg reflector centered at $885 \mathrm{~nm}$ at room temperature: (a) fixed monitoring wavelength of $633 \mathrm{~nm}$. (b) ideal monitoring conditions. (c) Reflectivity derivative variation for case (b). 


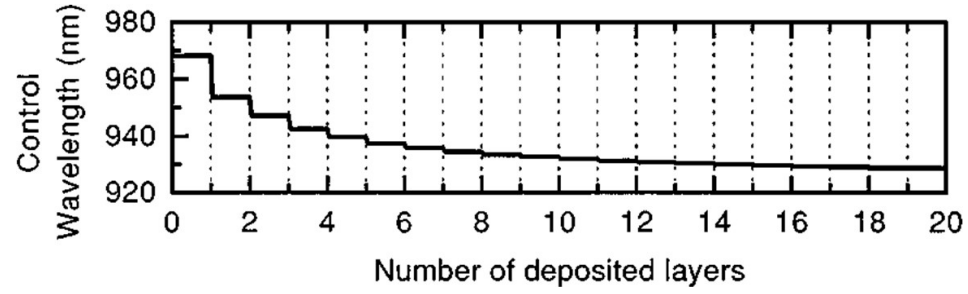

FIG. 2. Optimal wavelengths to be used for monitoring the successive layer thicknesses. 


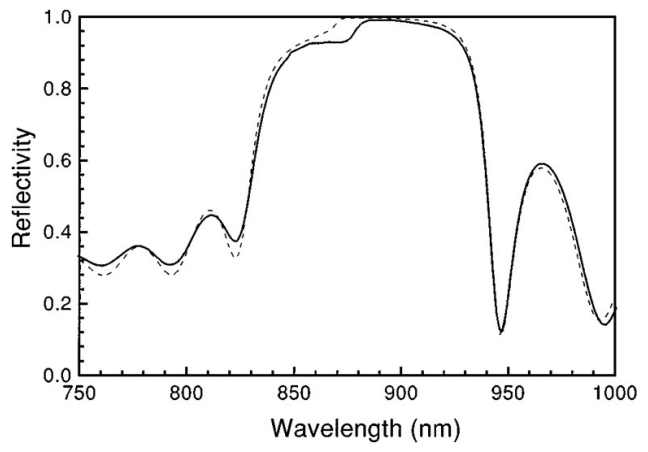

FIG. 3. Reflectivity spectra of 16-period GaAs-AlAs Bragg reflector at $885 \mathrm{~nm}$ : (a) modeling of the ideal Bragg reflector and (b) measurement of the multilayer structure grown under TDOR monitoring. 